\title{
LETTERS
}

\section{Urgent need to tackle Covid-19 impact on academic research in India.}

Published online first on January 9, 2021. DOI:10.20529/IJME. 2021.003

Keywords: Covid-19, lockdown, research misconduct, extended deadlines

The government of India imposed a strict nationwide lockdown on March 24, 2020, to arrest the spread of Covid-19 (1). Abiding by the government regulations, several educational institutions including those in healthcare, postponed or cancelled several academic activities to curb the spread of the virus (2). Considering the high risk of infection transmission, several academic research projects involving human participants were paused. This has posed serious challenges in managing academic tasks such as teaching and learning activities related to research, participant examination and follow-up, field work for data collection, face-to-face discussion with colleagues, students and supervisors. Many academic healthcare research activities involve human subjects as research participants; but because of the lockdown, final year post-graduate students may not be able to complete their research projects within the stipulated time frame. Healthcare students are in a vulnerable situation because of the pressure to submit their research projects for the successful completion of the courses. Given this situation, it is probable that students may resort to unethical or fraudulent research activities such as data manipulation or fabrication to complete their research projects before the existing deadline. Such research, if published in the future, can damage the credibility and validity of the scientific evidence.

Therefore, there is an urgent need to proactively evaluate this delicate situation, supervise, and recommend alternate best practices. The major responsibility rests now with medical universities and apex bodies such as the University Grants Commission, National Medical Council, Dental Council of India, Ministry of AYUSH, and Indian Council of Medical Research which directly or indirectly regulate academic research activities in the healthcare institutions of India.

Anticipating the high probability of fraud in academic research projects of healthcare students, we would like to make a few recommendations to tackle the situation.

- Universities and research regulating bodies should extend the dates for research projec $t$ submission. Already a few universities have issued a notice of extension of deadline for dissertation submission $(3,4)$.

- They should allow the submission of incomplete research projects (especially projects requiring human participants) for final year students who are on the verge of completion of their courses. This may lead to poor quality research submission, but it will increase transparency and reduce scientific misconduct in research projects. Though such research may not add any quality evidence to science, we should be happy that this will not harm science by publishing fraudulent research in the future.

- Universities should permit newly-admitted healthcare professional students to switch their research projects if they have already submitted the protocols and feel that it may not be possible to implement those projects in the near future, considering the uncertainty of the current pandemic situation.

These measures may not be sufficient, but are definitely necessary to reduce possible future scientific misconduct in academic research in India. We suggest that this serious issue should be debated and evaluated thoroughly by involving all stakeholders such as educationists, scientists, experts in research ethics, the relevant government authorities, policymakers, and finally healthcare students, to formulate strategies which are beneficial and also implementable.

These amendments will not only reduce the unwarranted pressure on students but will also help them in developing the sense of research integrity they need to practise in the future. We believe that, in this era, where we observe the prevalent scientific misconduct such as predatory publishing, retractions because of data manipulation, and other fraudulent practices which are very damaging to scientific evidence, we need competent researchers who are more aware of ethics and will practise research integrity.

Umesh Wadgave (corresponding author dr.w.umesh@gmail.com), Chief Dentist, Wadgave's Dental Home, House no 9-1-748, Pooja Nivas, Nandi Colony, Bidar, Karnataka, 585401 INDIA; Mahesh Khairnar (kmahesh222@gmail.com), Assistant Professor, Public Health Dentistry, Faculty of Dental Sciences, IMS BHU, Varanasi, UP, 221 005, INDIA

\section{References}

1. Press Information Bureau, Govt of Insia. MHA. Government of India issues Orders prescribing lockdown for containment of COVID Epidemic in the country.. New Delhi: Gol; 2020 Mar 24[cited 2020 Dec 28]. Available from: https://www.mha.gov.in/sites/default/files/ PR_NationalLockdown_26032020_0.pdf

2. Secretary, University Grants Commission. Preventive measures to achieve 'social distancing': Permission to teaching and non-teaching staff to work from home. 2020 Mar 21[cited 2020 Dec28]. Available from https://www.ugc.ac.in/pdfnews/7248697_UGC-Advisory--Permission-to-work-from-home-in-view-of-COVID-19.pdf

3. Rajiv andhi University of Health Sciences. Regarding extension of last date for submission of dissertation for Post Graduate students of Medical Courses during May.2021 examinations. 2020 Oct 17 [cited 2020 Dec 28]. Available from https://www.rguhs.ac.in/Exam/2020/ Extension $\% 20$ of\%20last $\% 20$ date $\% 20$ for\%20submission\%20of\%20dis sertation\%20for\%20PG\%20Medical\%20courses\%20May\%202021\%2 OExamination.pdf

4. Controller of Examinations, Maharashtra University of Health Sciences, Nashik. Letter dated November 29, 2020 [cited 2020 Dec 15]. Available from https://www.muhs.ac.in/showpdf.aspx?src1=upload/ Examination\%20Circular_6413_291120.pdf

\section{The Covid-19 uncertainty and ethical dilemmas in} dental practice

Published online on October 24, 2020. DOI: 10.20529/IJME. 2020.105 .

The Covid-19 pandemic has affected dental practice globally. Proximity with patients and predominance of aerosolgenerating procedures has raised concerns regarding the safety of dentists and patients alike. The near-total, yet inevitable, suspension of dental practice has raised several 